Research Paper

\title{
The Prostate Basal Cell (BC) Heterogeneity and the p63-Positive BC Differentiation Spectrum in Mice
}

\author{
Dong-Kee Lee ${ }^{1}$, Yonghong Liu ${ }^{1,2}$, Lan Liao ${ }^{1}$, Fen Wang ${ }^{2}$, and Jianming $\mathrm{Xu}^{1,3 凶}$ \\ 1. Department of Molecular and Cellular Biology, Houston, Texas, USA; \\ 2. Institute of Bioscience and Technology, Texas A\&M University Health Science Center, Houston, TX, USA; \\ 3. Institute for Cancer Medicine, Luzhou Medical College, Luzhou, Sichuan, China.
}

$\triangle$ Corresponding author: Jianming Xu, PhD, Department of Molecular and Cellular Biology, Baylor College of Medicine, One Baylor Plaza, Houston, Texas 77030. Tele: 713-798-6199; Fax: 713-798- 3017; E-mail: jxu@bcm.edu.

( ) Ivyspring International Publisher. This is an open-access article distributed under the terms of the Creative Commons License (http://creativecommons.org/ licenses/by-nc-nd/3.0/). Reproduction is permitted for personal, noncommercial use, provided that the article is in whole, unmodified, and properly cited.

Received: 2014.06.27; Accepted: 2014.07.27; Published: 2014.09.06

\begin{abstract}
The prostate epithelium is composed of basal (BC), luminal (LEC), and neuroendocrine (NEC) cells. It is unclear how many subtypes of BCs in the prostate and which subtype of BCs contains the main stem cell niche in the adult prostate. Here we report seven $\mathrm{BC}$ subpopulations according to their $\mathrm{p} 63$, cytokeratin $14 \quad(\mathrm{~K} / 4)$ and $\mathrm{K} 5$ expression patterns, including p63-positive/KI4-negative/K5-negative (p63+/KI4-/K5-), p63-/KI4+/K5-, p63-/KI4-/K5+, $\mathrm{p} 63+/ \mathrm{K} I 4+/ \mathrm{K} 5-, \mathrm{p} 63+/ \mathrm{K} / 4-/ \mathrm{K} 5+, \mathrm{p} 63-/ \mathrm{K} / 4+/ \mathrm{K} 5+$, and $\mathrm{p} 63+/ \mathrm{K} / 4+/ \mathrm{K} 5+\mathrm{BCs}$. We generated a p63-CreERT2 knock-in mouse line that expresses tamoxifen-inducible Cre activity in the p63-expressing cells, including the prostate BCs. We then crossbred this line with ROSA26R mice, and generated p63-CreERT2 $\times$ ROSA26R bi-genic mice harboring the Cre-activated $\beta$-galactosidase reporter gene. We treated these bi-genic mice with tamoxifen to mark the p63+ $\mathrm{BC}$ s at different ages or under different hormonal conditions, and then traced the lineage differentiation of these genetically labeled BCs. We discovered that these p63+ BCs contain self-renewable stem cells in culture and efficiently differentiated into LECs, NECs and BCs in the postnatal, adult and re-generating mouse prostates. Therefore, BC population contains heterogeneous $B C$ s that express different combinations of the $\mathrm{p} 63, \mathrm{KI} 4$ and $\mathrm{K} 5$ differentiation markers. Because $\mathrm{KI} 4+$ and $\mathrm{K} 5+\mathrm{BCs}$ were previously shown to be extremely inefficient to produce LECs in adulthood, we propose that the $\mathrm{p} 63+/ \mathrm{K} 5-/ \mathrm{K} / 4$ - subpopulation of $\mathrm{BCs}$ contains most stem-like cells, especially in adult animals.
\end{abstract}

Key words: Prostate epithelium, basal cell differentiation, p63, lineage tracing, stem cell.

\section{Introduction}

The prostate originates from epithelial budding from an intermediate region of the primitive urogenital sinus, which is a hindgut derivative of the endoderm. Both human and murine prostate glands are composed of an outer layer of basal cells (BCs), which lie on a basement membrane, and an inner layer of luminal epithelial cells (LECs). The neuroendocrine cells (NECs) are rare in number and are scattered among LECs without extending to the lumen [1]. LECs express cytokeratin 8 (K8), K18 and androgen receptor (AR). BCs express K14, K5 and p63. NECs express synaptophysin (Syp), somatostatin and chromogranin A and also possess numerous rich serotonin-containing granules [2].

Lineage tracing studies during prostate development and homeostasis are relevant to the origin of prostate cancer cells. In vitro and ex vivo studies have suggested that subsets of BCs in adult murine prostate may contain pluripotent and self-renewal stem cells, which can be related to prostate cancer initiation $[3,4]$. Similarly, a subset of human prostate BCs is able to reconstitute prostatic gland in a model system [5], and 
tracing the cell lineage with mitochondrial DNA mutation also suggests that human prostate BCs may contain stem cells to generate other types of epithelial cells in the prostate [6, 7]. However, recent prostate epithelial lineage-tracing studies using genetically manipulated mouse models have provided different results regarding the multi-potent capabilities of BCs and LECs to generate other types of epithelial cells in the prostate. Although the K14-positive $(\mathrm{K} 14+)$ BCs were shown to be able to differentiate into other types of epithelial cells during postnatal prostate morphogenesis, genetically marked $\mathrm{K} 14+\mathrm{BCs}$ in the adult prostate were unable to generate LECs [8, 9]. Furthermore, genetically marked $\mathrm{K} 5+\mathrm{BC}$ in the prostate of adult mouse could produce very few, if any, LECs under normal physiological conditions $[9,10]$. These findings indicate that the $\mathrm{K} 14+$ or $\mathrm{K} 5+\mathrm{BCs}$ in the prostate of adult animal are unlikely the multipotent tissue stem cells. Furthermore, prostatic BCs in the adult animal have not been shown to be able to generate NECs. Hence, it remains to be addressed whether the prostatic BC population in the adult animal contains stem cells with a full differentiation spectrum.

Here, we report the identification of seven BC

A
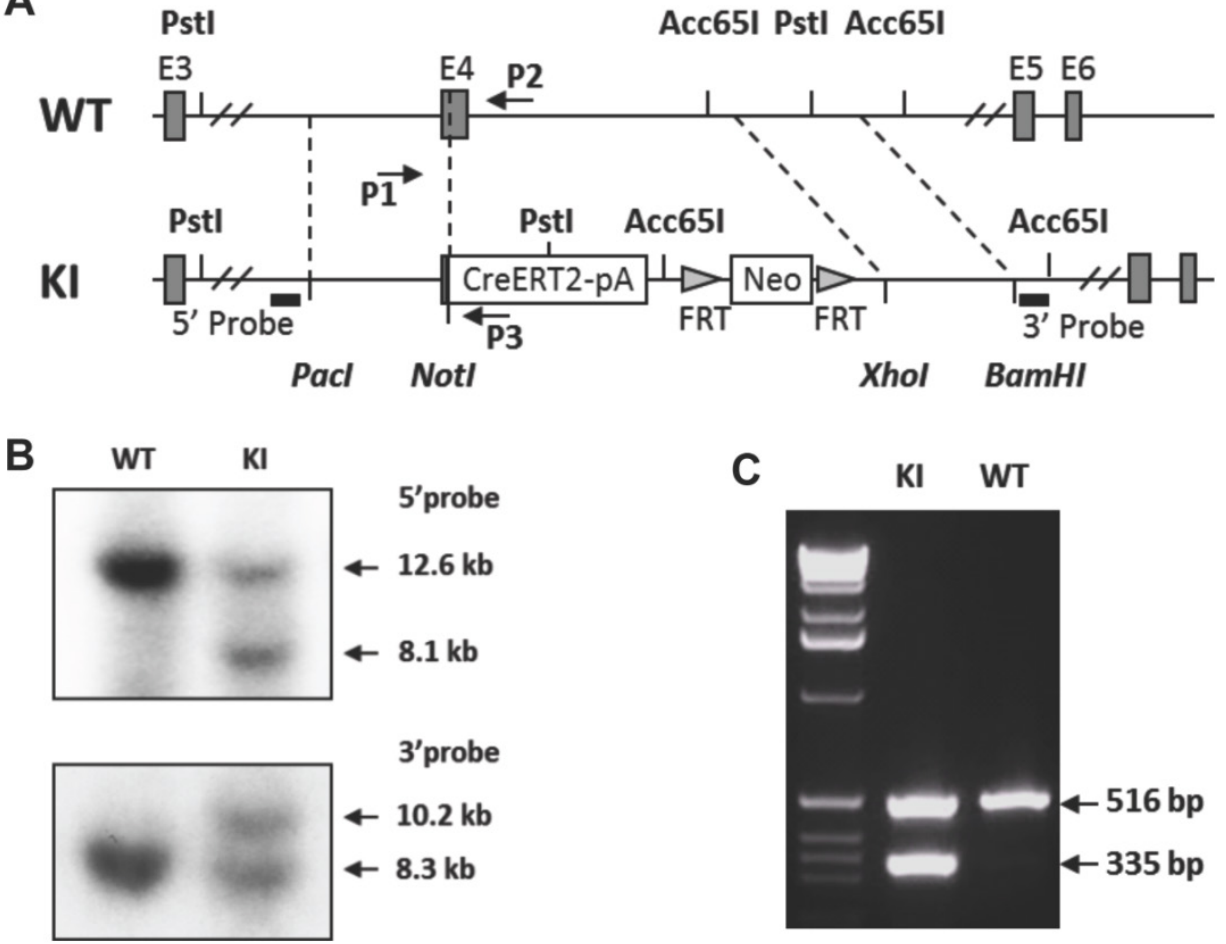

Figure 1. Generation of the p63-CreERT2 knock-in mouse line. A. Schematic drawing of the murine p63 gene wild type (WT) allele and the CreERT2-pA-FRT-Neo-FRT knock-in (KI) allele. Exons (E) 3-6 are indicated in both alleles. The 5' and 3 ' homologous regions used in the targeting vector are indicated by dotted lines between WT and KI alleles. The locations of the 5' and 3' probes used in Southern blot are indicated. The locations of primers PI, P2 and P3 used for genotype analysis are also indicated. B. Southern blot analysis of the WT and KI alleles. The 5' probe-detected 12.6 and 8.1 kb fragments were respectively derived from the WT and KI alleles after Pst I digestion. The 3' probe-detected 10.2 and $8.3 \mathrm{~kb}$ fragments were respectively derived from the $\mathrm{KI}$ and $\mathrm{WT}$ alleles after Acc65 I digestion. C. Genotype analysis of mouse genotypes by PCR Mouse genomic DNA was used as PCR template. The 516 bp fragment was detected from the WT allele by primers PI and P2. The 335 bp fragment was detected from the $\mathrm{KI}$ allele by primers $\mathrm{PI}$ and $\mathrm{P} 3$. subpopulations in the mouse prostate, including the previously unrecognized p63+/K14-negative (K14-)/K5- BC subpopulation. We show that p63-expressing BCs contain multipotent stem cells that can efficiently differentiate into LECs, BCs and NECs during postnatal prostate morphogenesis, adult prostate epithelial homeostasis, and androgen-stimulated prostate epithelial regeneration in the adult animal.

\section{Materials and Methods}

\section{Mice}

The p63-CreERT2 mouse line was generated by a knock-in strategy illustrated in Figure 1. To construct the targeting vector, the genomic DNA of TC-1 mouse ES cells was used as PCR template for amplifying targeting arms. The $5^{\prime}$ targeting arm was amplified by PCR using primers $5^{\prime}$ - gcgttaattaaCTCTTAGTCCTGG ACATCCGCATTGAAGT with a Pac I site and 5' atagcggecgCCCCAGGTTCGTGTACTGTGGCTGT with a Not I site. The DNA coding CreERT2 and $\beta$-globin poly(A) addition-signaling sequence was amplified by PCR using primers $5^{\prime}$ - atagcggccgctg TCCAATTTACTGACCGTACACC with a Not I site and $5^{\prime}$ acaggtaccCTTCGAATTCCT CGACCAGAC with an Acc65 I site. These two PCR products were cloned into the targeting vector between Pac I and Acc65 I sites, which were upstream of the FRT-flanked PGK-neo cassette. The 3'-targeting arm was amplified by PCR using primers 5' acgctcgagCCGGAGCAATC AACTTTGAAGACAGTAC with an Xho I site and 5'agcggattcGGAAAGTACCA ACAGCAAATATCCCACA G with an EcoR I site. The 3' arm was cloned into the targeting vector between PGK-neo and HSV-tk cassettes. The vector was linearized by Pac I digestion. The vector was designed to knock in the CreERT2-coding sequence into exon 4, resulting in an in-frame fusion after the $115^{\text {th }}$ a.a. of p63 or the $24^{\text {th }}$ a.a. of $\Delta \mathrm{Np} 63$ isoform (Fig. 
1A). The TC-1 mouse ES cells were transfected with the vector DNA by electroporation, cultured on feeder cells, and selected in medium containing G418 and fialuridine (FIAU) as described previously [11, 12]. Survived individual colonies were isolated and screened by Southern blotting. The $5^{\prime}$ probe was used to detect the $12.6-\mathrm{kb}$ and $8.1-\mathrm{kb}$ fragments produced from the respective $5^{\prime}$ regions of the wild type and targeted alleles after Pst I digestion. The $3^{\prime}$ probe was used to detect the $8.3-\mathrm{kb}$ and $10.2-\mathrm{kb}$ fragments were produced from the respective 3 ' regions of the wild type and targeted alleles after Acc65 I digestion (Fig. 1B). The targeted clones were injected into C57BL/ 6 blastocysts to produce chimeric mice. Male chimeric mice were further crossbred with female FLPeR mice [13] to remove the Frt-flanked PGK-neo cassette and simultaneously obtain the p63-CreERT2 heterozygous mouse line as described previously [12]. Mouse genotype analysis was carried out by PCR using DNA template prepared from a piece of ear tissue and three primers. Primers P1 (5'-AATGTTGGGGTGTCTGGA

TG) and P2 (5'-CAGCAGTCAGGAACAAAGAGG) were used to detect the 516-bp fragment of the $p 63$ wild type allele. Primers P1 and P3 (5'-GCCCAAATGTTGCTGGATAG) were used to detect the 335-bp fragment of the p63-CreERT2 knock-in allele (Fig. 1C).

The ROSA26R mice harboring a Cre-activated expression of the $\beta$-galactosidase ( $\beta$-gal) reporter gene [14] were obtained from the Jackson Laboratory. The bi-genic mice used in this study were generated by crossbreeding ROSA26R mice with p63-CreERT2 mice. Animal protocols were approved by the Institutional Animal Use and Care Committee at Baylor College of Medicine.

\section{Animal treatment}

Tamoxifen (Sigma Chemical Co., St. Louis, MO) was dissolved in corn oil at $20 \mathrm{mg} / \mathrm{ml}$. Mice were treated with tamoxifen $(100 \mathrm{mg} / \mathrm{kg} /$ day, i.p.) for five consecutive days for inducible activation of the $\beta$-gal reporter expression. In some experiments, mice were castrated at 8 weeks of age, treated with tamoxifen at 10 weeks of age and then with a testosterone pellet (5 $\mathrm{mg} /$ pellet in Beeswax, Sigma) at 12 weeks of age, followed by a replacement of the pellet with a fresh one at 14 weeks of age. Their prostates were examined at 13 and 16 weeks of ages.

\section{$X$-gal staining and immunofluorescence}

For X-gal staining, prostate tissue was fixed with $2 \%(\mathrm{w} / \mathrm{v})$ paraformaldehyde (PFA) for 1 hour, washed with PBS for 1 hour, incubated in an incubation buffer for 1 hour, and then incubated with X-gal-staining solution for 16 hours at room temper- ature. The stained tissue was dehydrated, paraffin-embedded, sectioned and counter-stained with Nuclear Fast Red. For immunofluorescence, rehydrated prostate tissue was boiled in a citrate buffer (pH 6.0) for antigen retrieval, treated with 3\% hydrogen peroxide for inactivating endogenous peroxidase, washed with PBS containing $0.05 \%$ Tween-20, and blocked with $5 \%$ normal goat serum for 2 hours. For double immunofluorescent staining, the blocked tissue slides were incubated with the antibody against K5 (rabbit polyclonal, Covance, Princeton, NJ), K8 (rat monoclonal, Developmental Studies Hybridoma Bank, Iowa City, IA) or Syp (rabbit polyclonal, Invitrogen, Camarillo, CA) overnight at $4^{\circ} \mathrm{C}$, and with an appropriate fluorescence-labeled (Invitrogen) or biotinylated (Jackson ImmunoResearch Lab, West Grove, PA) secondary antibody for 1 hour at room temperature. Signals were amplified with the TSA-Alexa Fluor 488 kit (Invitrogen). Then, the tissue slides were incubated with the anti- $\beta$-gal antibody (chicken polyclonal, Abcam, Cambridge, MA) overnight at $4^{\circ} \mathrm{C}$ and the biotinylated secondary antibody for 1 hour at room temperature. Signals were amplified with the TSA-Alexa Fluor 594 kit (Invitrogen). For Triple immunofluorescent staining, the blocked tissue slides were incubated with antibodies against p63 (mouse monoclonal, Santa Cruz Biotechnology, Santa Cruz, CA), K14 (chicken polyclonal, Covance, Princeton, NJ) and $\mathrm{K} 5$ (rabbit polyclonal, Covance) overnight at $4^{\circ} \mathrm{C}$, and the biotinylated secondary antibodies for 1 hour at room temperature. Signals were amplified with the TSA-kit (Invitrogen). The Alexa-Fluor dyes used for p63, K5 and K14 were 594, 488 and 680, respectively.

\section{Results}

KI4, K5 and p63 triple immunofluorescent staining identified seven subtypes of $B C s$ including the subtype of p63+/K I 4-/K5- BCs.

It has been reported that LECs in adult mice are rarely derived from tamoxifen-inducible K14-CreERT2-marked BCs and also very inefficiently derived from tamoxifen-inducible K5-CreERT2marked BCs [8-10]. The discrepancy between these reports and the previous indirect evidence supporting the stem cells within the basal layer in adult mice [15] suggests a possible existence of K14-/K5- stem cells within the basal layer. To examine BC heterogeneity, we analyzed the co-expression patterns of the p63, K14 and K5 BC markers in the mouse prostate sections by triple immunofluorescent staining. Interestingly, we identified seven subtypes of BCs with different percentages in cell number, including p63+/K14-/K5- (3.2\%), p63-/K14-/K5+ (5.6\%), p63+/K14-/K5+ (4.0\%), p63-/K14+/K5- (1.6\%), 
p63+/K14+/K5- (9.6\%), p63-/K14+/K5+ (14.6\%) and p63+/K14+/K5+ (61.4\%) BCs (Fig. 2). These results indicate that individual $\mathrm{BCs}$ are heterogeneous and express different $B C$ markers and that different $B C$ subpopulations may have different structural and functional contributions to normal and cancerous prostates.

\section{The p63+ BCs in adult prostate contain epithelial stem cells.}

Identification of the previously unknown BC subpopulations provoked our interest in testing whether the p63+/K14-/K5- BC subpopulation contains multi-potent stem cells in the murine prostate. This subpopulation has not yet been tested in previ- ous studies tracing the $\mathrm{K} 5+$ or $\mathrm{K} 14+\mathrm{BC}$ lineages. To examine the lineage fate of the p63+ BCs, we constructed a knock-in vector, inserted the CreERT2-coding DNA for the tamoxifen-inducible Cre recombinase into the fourth exon of the $p 63$ gene in the mouse embryonic stem (ES) cells, and generated p63-CreERT2 mice from the targeted ES cells (Fig. 1). We crossed p63-CreERT2 mice with Rosa26R mice harboring a Cre-activated expression of the $\beta$-gal reporter [14] and generated p63-CreERT2 $\times$ Rosa26R bi-genic mice. In these bi-genic mice, the CreERT2 fusion protein expressed in p63+ BCs and remained inactive in the cytoplasm and thus, the $\beta$-gal reporter was silent before tamoxifen treatment.
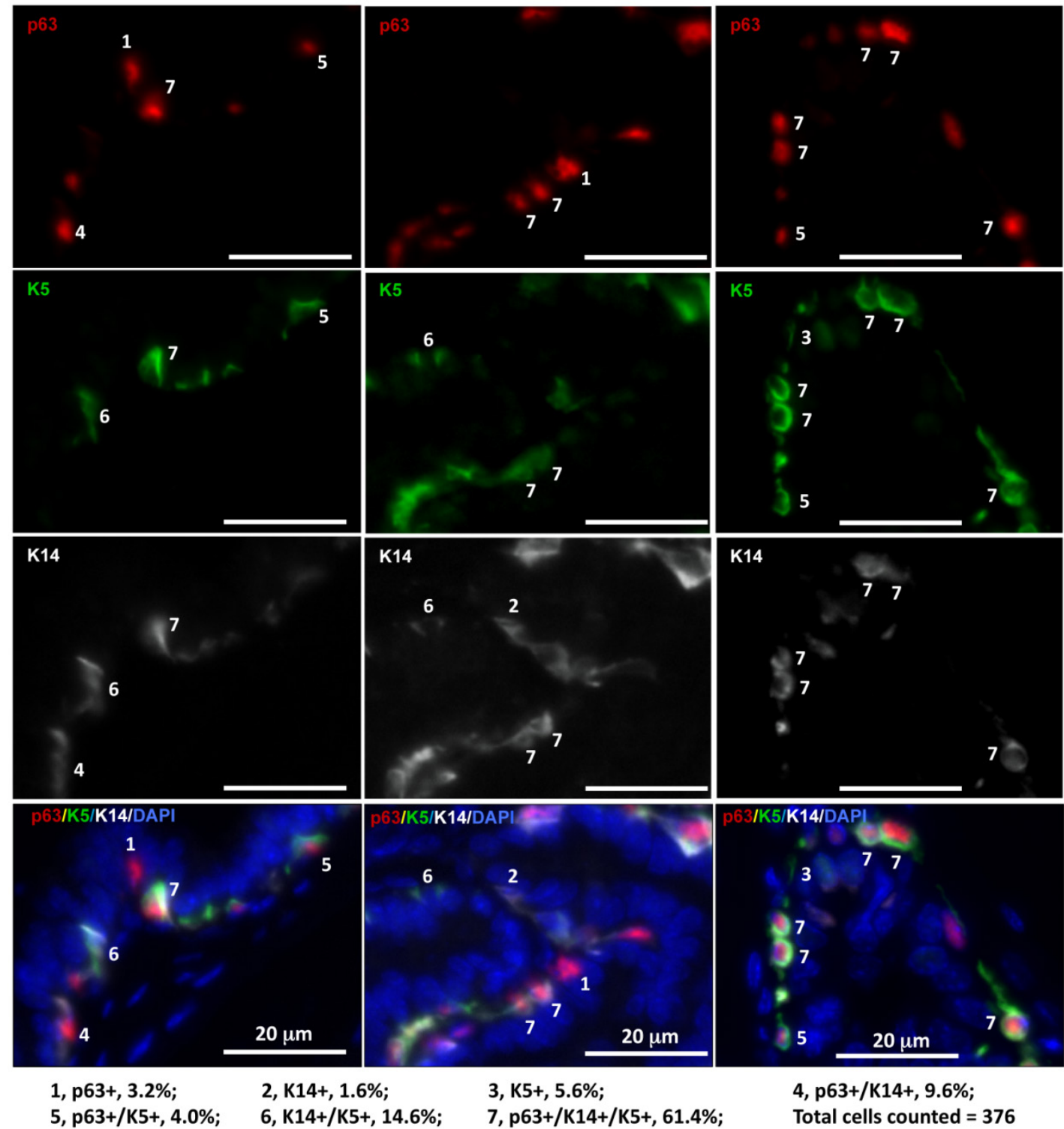

2, K14+, 1.6\%;

3, $\mathrm{K} 5+, 5.6 \%$;

6, K14+/K5+, 14.6\%

7, p63+/K14+/K5+, 61.4\%;

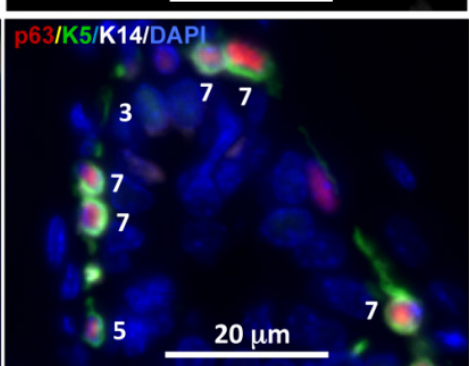

4, p63+/K14+, 9.6\%;

Total cells counted $=\mathbf{3 7 6}$

Figure 2. Identification of seven subtypes of BCs in the adult mouse prostate by triple immunofluorescent staining of p63, K5 and KI4. Triple immunofluorescent staining was performed on prostate sections prepared from three 6-week-old mice. Immunofluorescent staining signals of p 63 , K5 and KI 4 were separately imaged by using appropriate filters and a monochrome CCD camera and respectively labeled as red, green and white colors. Each of the three bottom panels is the merged image of the three panels above plus the blue DAPI staining signal for nuclei. The $7 \mathrm{BC}$ subtypes were distinguished according to the expression patterns of p63, K5 and KI4 as indicated. The percentage of each subtype $\mathrm{BCs}$ was calculated by normalizing to the total number of counted BCs. 
To examine whether p63+ BCs contain epithelial stem-like cells in the developed prostate, we treated 4-week-old p63-CreERT2 $\times$ Rosa26R mice with tamoxifen for 5 days to activate $\beta$-gal expression in the p63+ BCs. We confirmed that only a proportion of BCs but no LECs were positive to X-gal staining in one week after tamoxifen treatment (Fig. 3A and B). At this time point, we isolated prostate epithelial cells and performed epithelial sphere formation assay in Matrigel culture. From the initial culture, about 200 spheres in average were developed from epithelial cells isolated from each mouse. About $48 \%$ and $52 \%$ of these spheres composed of $\beta$-gal-negative ( $\beta$-gal-) and $\beta$-gal-positive ( $\beta$-gal+) cells, respectively (Fig. $3 \mathrm{~A}$ and $C)$. The $\beta$-galt spheres were derived from the p63-CreERT2-marked BCs. We subsequently digested all spheres into single cell suspension and re-cultured these cells for developing the second generation of spheres, followed by serial passages of third and fourth generations of sphere formation assays. We found that about $30 \%$ of the spheres composed of only $\beta$-gal+ cells throughout these passages (Fig. 3A). The lower percentage of $\beta$-gal+ spheres in the second generation versus the first generation suggests that some initial $\beta$-gal+ spheres were developed from partially differentiated, non-self-renewable intermediate p63+ BCs. We also isolated prostate epithelial cells from 6-week-old mice without tamoxifen treatment

A p63-CreERT2 $\times$ Rosa26R Mice

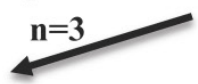

Day 28-32: +Tam

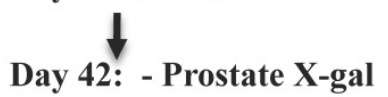
Staining (Panel B)

- G1 sphere assay

(Panel C, 52\% BSs)

G2 sphere assay (29\% BSs)

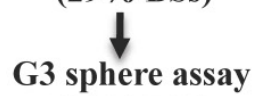
(28\% BSs)

G4 sphere assay (27\% BSs)

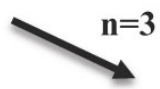

Day 42: G1 sphere growth

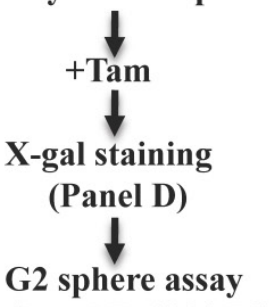

(Panel E, 13\% BSs)

G3 sphere assay

G4 sphere assay

(13\% BSs)
(14\% BSs) and allowed them form epithelial spheres in culture (Fig. 3A). After a brief tamoxifen treatment in culture, one to several cells in a sphere were stained blue by X-gal staining (Fig. 3D), suggesting that these were p63+ cells in these spheres. After the cells of these spheres were assayed in serial passages, we again identified a constant ratio of $13-14 \%$ blue spheres throughout the subsequent passages (Fig. $3 \mathrm{~A}$ and E). These results clearly indicate that the p63+ BCs in the adult mouse prostate contain self-renewable stem-like cells.

\section{The p63-expressing BCs contain pluripotent stem-like cells during postnatal prostate morphogenesis.}

To trace the lineage differentiation of the p63-expressing BCs during postnatal development, we treated 2-week-old p63-CreERT2 $\times$ Rosa26R mice with tamoxifen, collected prostates 1 and 4 weeks after tamoxifen treatment, and performed X-gal staining (Fig. 4A). One week after tamoxifen administration, X-gal staining signal was only observed in BCs (Fig. 4B). Double immunofluorescent staining revealed that $27.6 \%$ of $\mathrm{K} 5+\mathrm{BC}$ were also $\beta$-gal positive (Fig. $4 \mathrm{D}$ and $\mathrm{G}$ ), while no $\beta$-gal+/K8+ LECs and $\beta$-gal+/Syp+ neuroendocrine cells (NECs) were observed at this time point (Fig. 4E and G, and data not shown).

Figure 3. The p63+ BCs in the developed mouse prostate contain epithelial stem-like cells. A. Experimental flowchart. Three mice were used for each experiment $(n=3)$. GI-G4 indicate sphere generations I-4. The percentages of blue spheres (BSs), which are the $\beta$-gal+ spheres derived from the marked $\mathbf{p} 63+\mathrm{BCs}$, are indicated. B. X-gal-stained prostate tissues prepared from vehicle or tamoxifen-treated p63-CreERT2 $\times$ Rosa26R mice. C. X-gal-stained epithelial spheres formed from isolated prostate epithelial cells of tamoxifen-treated p63-CreERT2 $\times$ Rosa26R mice. White (WS) and blue (BS) spheres are indicated. D. A tamoxifen-treated epithelial sphere developed from the isolated prostate epithelial cells of untreated $\mathrm{p} 63-\mathrm{CreERT2} 2 \times$ Rosa26R mice. E. A blue second-generation sphere developed from re-culturing cells of the tamoxifen-treated first-generation spheres. 


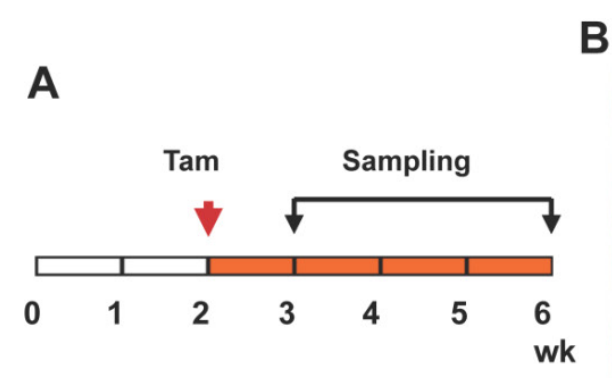

B 1 wk after Tam

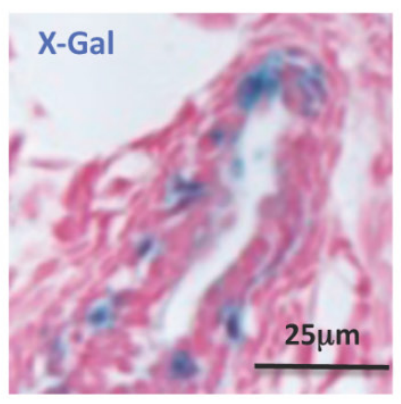

C 4 wk after Tam

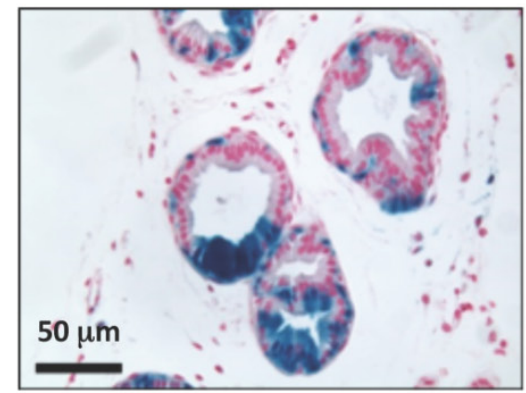

\section{D}
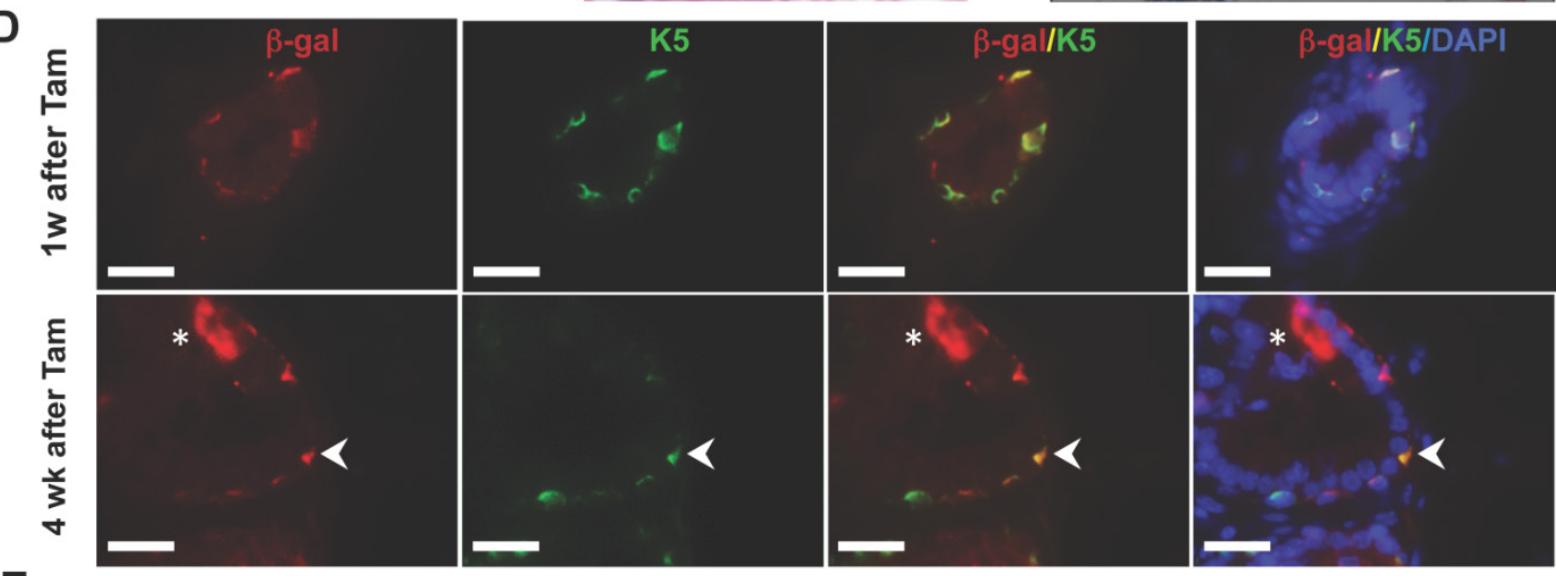

E
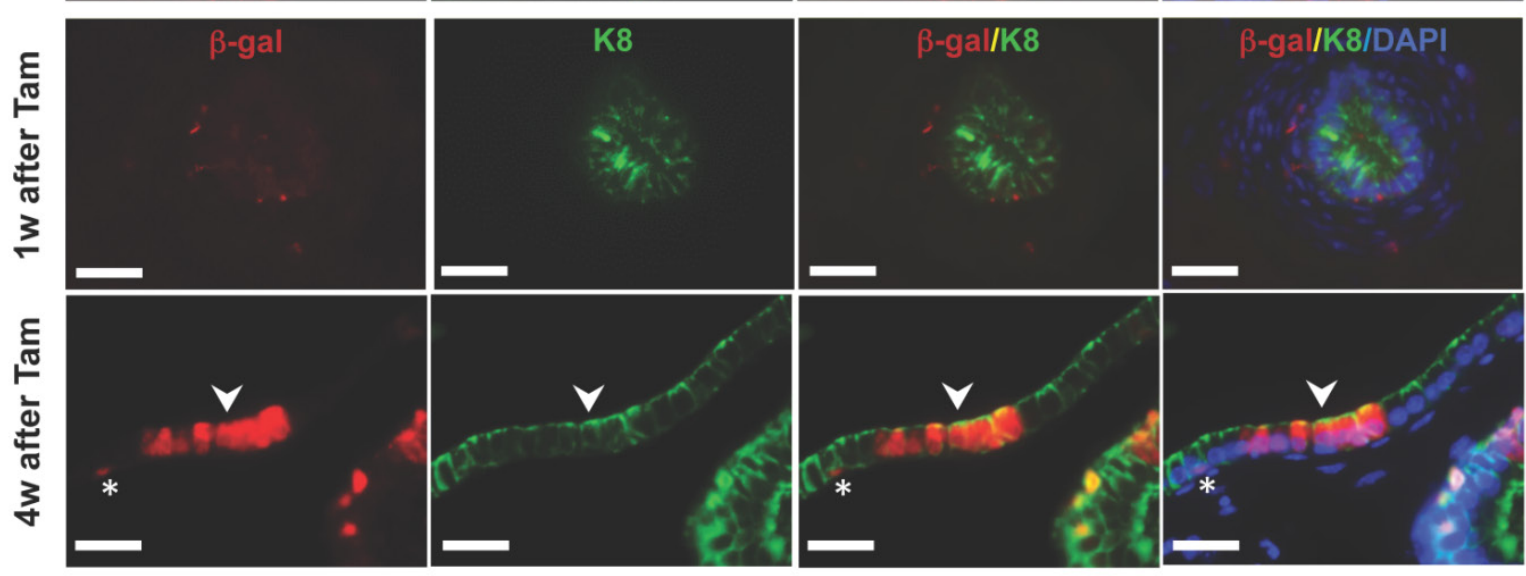

F
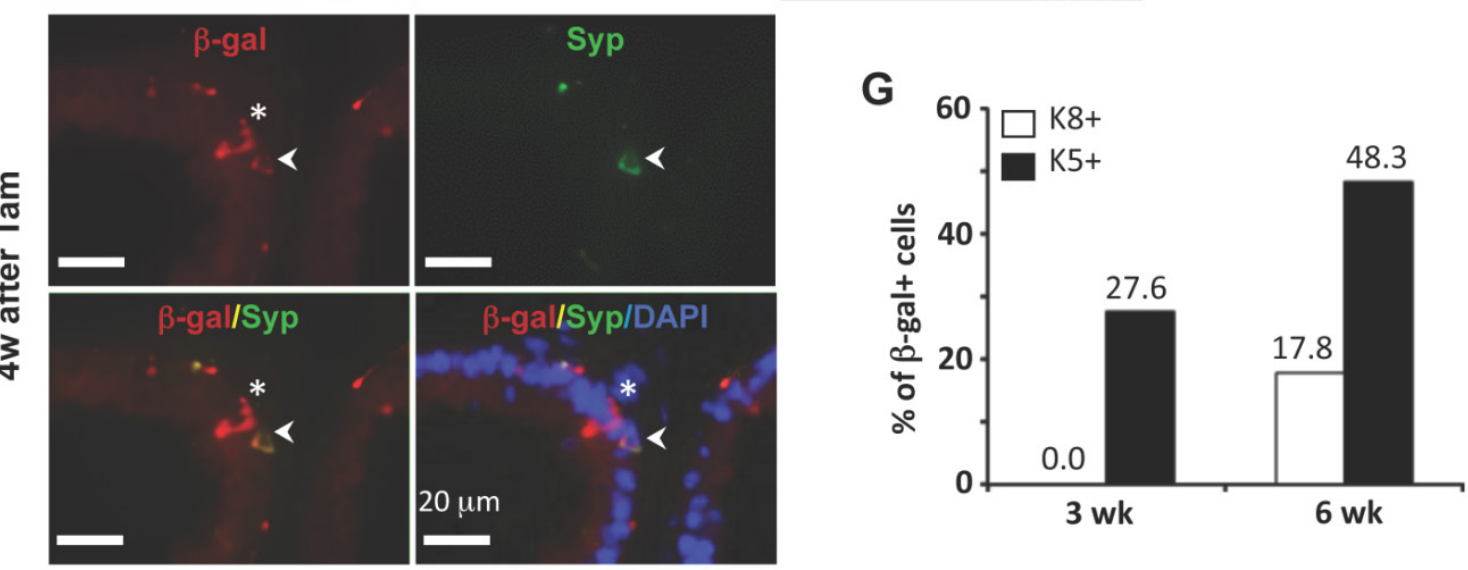

Figure 4. LECs, BCs and NECs developed from p63+ BCs during postnatal prostate morphogenesis. A. Schematic of the lineage tracing experiment during postnatal prostate development of p63-CreERT2×Rosa26R mice. B and C. X-gal-stained prostate tissues at 3 and 6 weeks of age. The blue signal indicates $\beta$-gal-expressing cells in basal (panel B) and in both basal and luminal layers (panel C). D and E. Unmerged and merged images of double immunofluorescent staining for $\beta$-gal and K5 (panel D) and for $\beta$-gal and K8 (panel E) on prostate sections at I and 4 weeks after tamoxifen treatment. Arrowheads indicate double positive cells. Asterisks indicate a $\beta$-gal+/K5- LEC in panel $\mathrm{D}$ and a $\beta$-gal+/K8- BC in panel E. F. Unmerged and merged images of double immunofluorescent staining for $\beta$-gal and Syp on prostate sections at 4 weeks after tamoxifen treatment. The arrowhead indicates a $\beta$-gal+/Syp + NEC, which is rare in number. Asterisks indicate two $\beta$-gal+/Syp- BCs and one LEC. G. The percentage of $\beta$-gal+/K8+ to total $\mathrm{K} 8+$ cells and the percentage of $\beta$-gal $+/ \mathrm{K} 5+$ cells to total $\mathrm{K} 5+$ cells at 3 and 6 weeks of ages. 
However, 4 weeks after tamoxifen treatment, $\beta$-gal+ LECs were clearly detected (Fig. 4C). Double immunofluoresence staining identified $48.3 \%$ of K5+ BCs, $17.8 \%$ of K8+ LECs, and certain Syp+ NECs as $\beta$-gal+ cells in the prostates of p63-CreERT2 $\times$ Rosa26R mice (Fig. 4D-G, and data not shown). These results indicate that the p63-expressing BCs in the prostate of 2-week-old mouse contain pluripotent stem-like cells that can generate LECs, BCs and NECs at the postnatal stage of prostate morphogenesis. One previous study also showed that isolated p63+ BCs could generate other types of prostate epithelial cells in vitro [16], while another study used a non-inducible mouse model to follow the fate of p63+ cells since embryo development and suggested p63+ cells as the prostate progenitor cells [17]. Together, these findings support the claim that p63+ BCs contain epithelial stem cells during postnatal morphogenesis of the prostate. Interestingly, the K14+ BCs were also shown to be able to develop into all types of prostate epithelial cells at this early postnatal developmental stage of prostate although these cells were unable to do so in adulthood $[8,9]$, suggesting that the stem cell population at this stage is different from that in the adult prostate.

\section{The p63-expressing BCs generate LECs, BCs and NECs during maintenance of the epithelial homeostasis of the prostate in adult mouse.}

Identification of stem cells in the adult prostate is extremely valuable because these cells may serve as prostate cancer-initiating cells in the adult prostate. To investigate whether p63+ BCs in the adult prostate contain epithelial stem cells, we treated 8-week-old p63-CreERT2 $\times$ Rosa26R mice with tamoxifen to mark BCs with $\beta$-gal expression and assessed $\beta$-gal+ cell types by X-gal staining and double immunofluorescent staining 1 or 4 weeks after tamoxifen treatment (Fig. 5A). At the 1-week time point, all $\beta$-gal+ cells were located in the basal layer (Fig. 5B). Most of these $\beta$-gal+ cells were $\mathrm{K} 5+\mathrm{BC}$, but none of these $\beta$-gal+ cells were K8+ LECs or Syp+ NECs (Fig. 5D and E, and data not shown). However, at the 4-week time point, $\beta$-gal+ LECs cells were clearly observed (Fig. 5C). Double immunofluorescent staining not only identified gal $+/ \mathrm{K} 5+$ BCs but also identified $\beta$-gal+/K8+ LECs as well as the gal+/Syp+ NECs (Fig. 5D-F). These results demonstrate, for the first time, that $\mathrm{p} 63+\mathrm{BCs}$ in the adult prostate can generate all three types of prostatic epithelial cells including the rare NECs.

To determine the dynamic contributions of the p63+ BCs to the prostate epithelial homeostasis over a long period of time, we treated young adult
p63-CreERT2 $\times$ Rosa26R mice with tamoxifen for 7 days (2 days longer than other experiments to enhance efficiency), and isolated their prostates for X-gal staining at 4, 8, 12 and 20 weeks after tamoxifen treatment. Interestingly, about $80-85 \%$ of BCs were stained positive to $\beta$-gal expression throughout all time points, suggesting that these p63+ BCs maintain their homeostasis through self-renewal. More strikingly, the percentages of $\beta$-gal+ LECs were progressively increased from $2 \%$ at the $4^{\text {th }}$ week to $43 \%$ at the $20^{\text {th }}$ week after tamoxifen treatment (Fig. 5G). These results demonstrate, for the first time, that the p63+ $\mathrm{BCs}$ in the adult mouse prostate can efficiently generate new LECs to maintain the prostate epithelial homeostasis.

\section{The p63-expressing BCs efficiently produce LECs and NECs during prostate regeneration.}

In the castration-testosterone $(\mathrm{T})$ replacement mouse model, the prostate epithelium experiences a highly dynamic remodeling process involving apoptosis, proliferation and differentiation, making it an ideal model for tracing BC differentiation into other types of epithelial cells. We castrated 8-week-old p63-CreERT2 $\times$ Rosa26R mice, then treated these mice with tamoxifen for 5 days at 10 weeks of age, and subsequently with $\mathrm{T}$ from 12 to 16 weeks of age (Fig. $6 \mathrm{~A})$. In the prostates of castrated mice before and after $\mathrm{T}$ treatment for one week, $\beta$-gal expression was only observed in BCs (Fig. 6B and C). However, after T treatment for 4 weeks, $\beta$-gal expression was detected in many of both BCs and LECs (Fig. 6D). Double immunofluorescent staining confirmed that $\beta$-gal $+/ \mathrm{K} 5+$ BCs but no $\beta$-gal+/K8+ LECs were observed at the time points before or after $\mathrm{T}$ treatment for one week (Fig. 6E and $\mathrm{F}$, and data now shown). After $\mathrm{T}$ treatment for 4 weeks, both $\beta$-gal $+/ \mathrm{K} 5+\mathrm{BCs}$ and $\beta$-gal+/K8+ LECs were observed (Fig. $6 \mathrm{E}$ and F). Quantitative analysis revealed that the percentage of $\beta$-gal+/K5+ BCs versus total BCs was significantly increased over time upon $\mathrm{T}$ treatment, while a significant percentage of $\mathrm{p} 63+/ \mathrm{K} 8+$ LECs were only detected after 4 weeks of $\mathrm{T}$ treatment (Fig. 6G). Interestingly, the $\beta$-gal+/Syp+ NECs were observed both before and after $\mathrm{T}$ treatment for 1 and 4 weeks (Fig. 7), suggesting an androgen-independent differentiation process from BCs to NECs in the adult prostate. These results demonstrate that $\mathrm{p} 63+\mathrm{BC}$ population contains stem cells that can differentiate into all three types of prostate epithelial cells during prostate regeneration in response to $\mathrm{T}$ stimulation. 
A

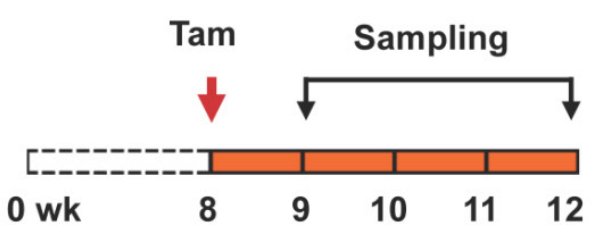

B 1 wk after Tam

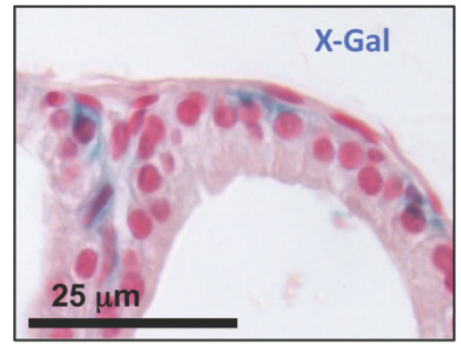

C 4 wk after Tam

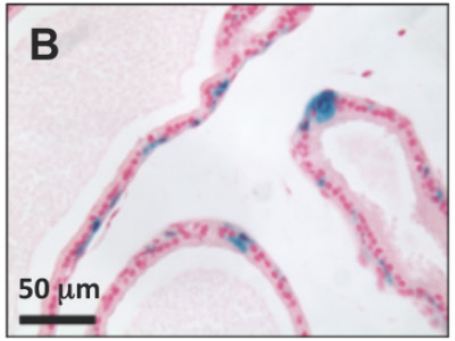

D
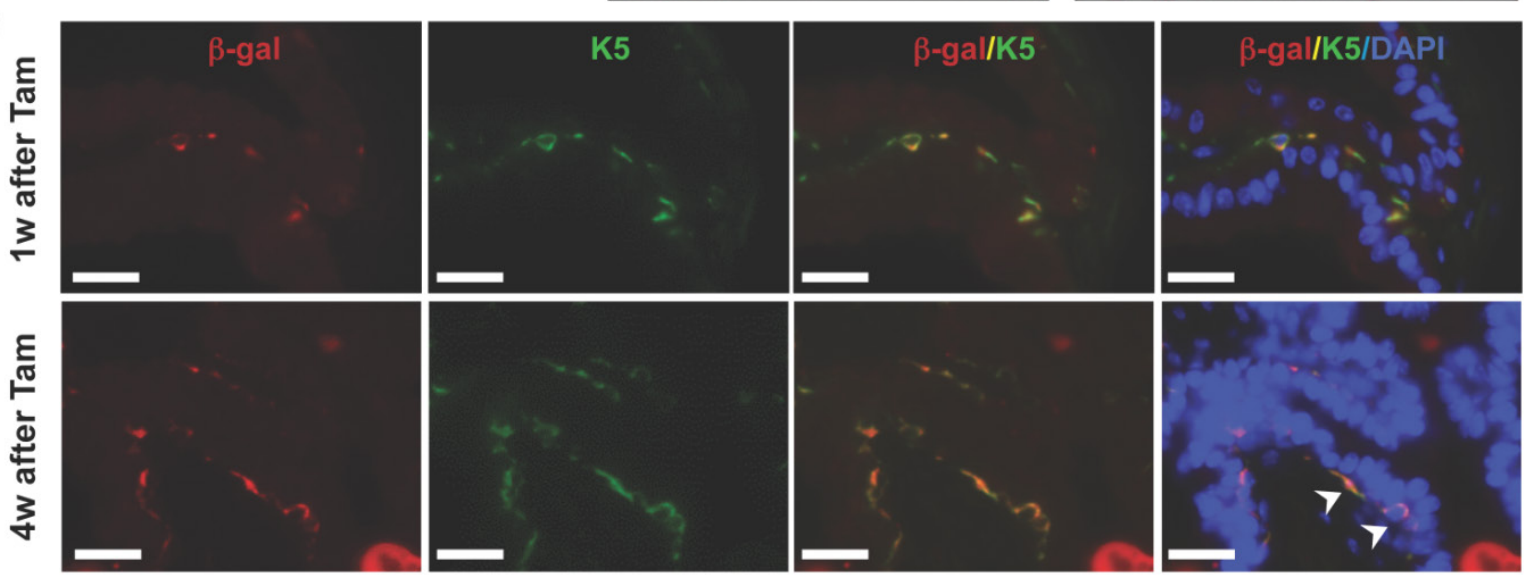

E
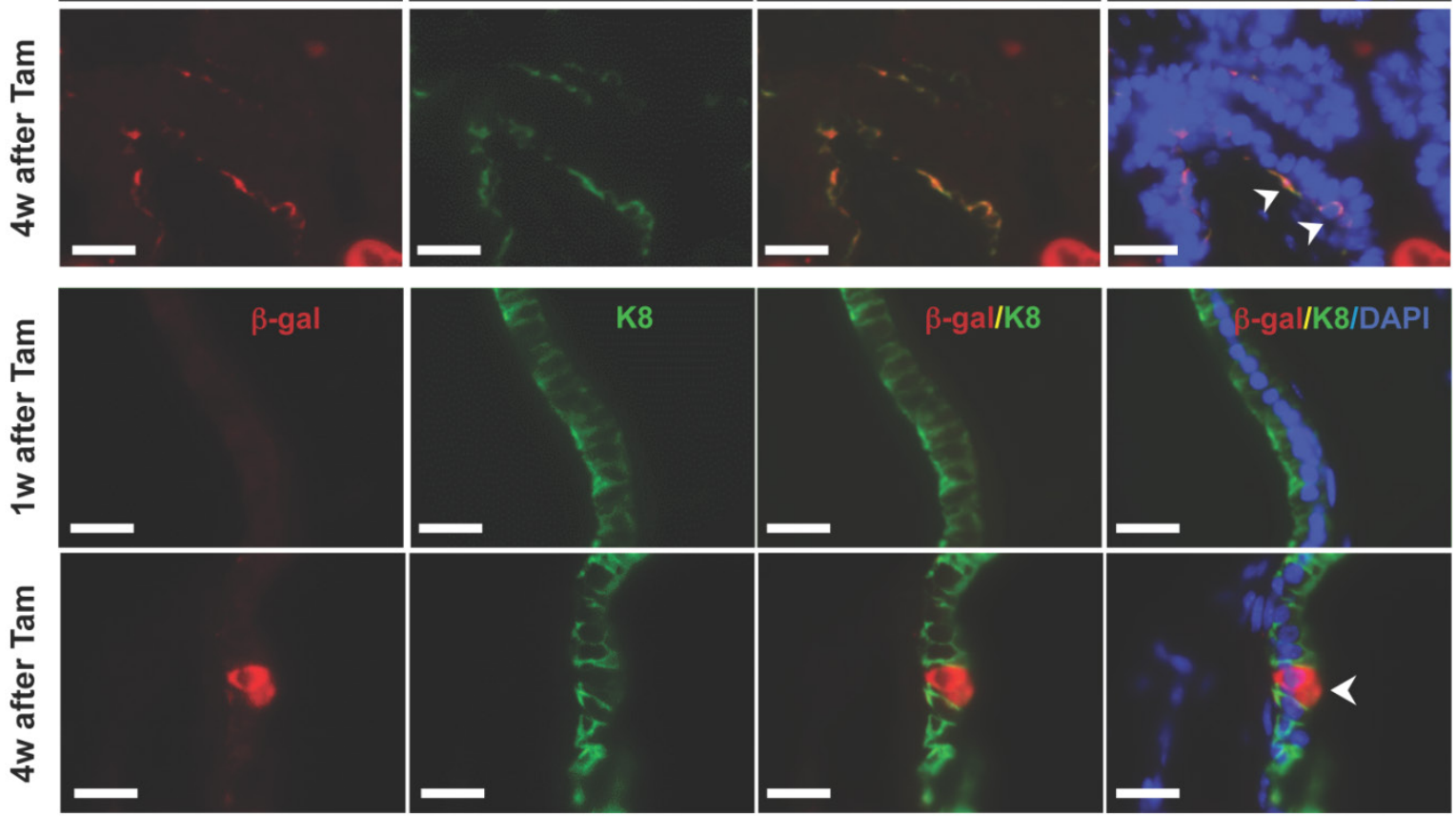

$\mathbf{F}$
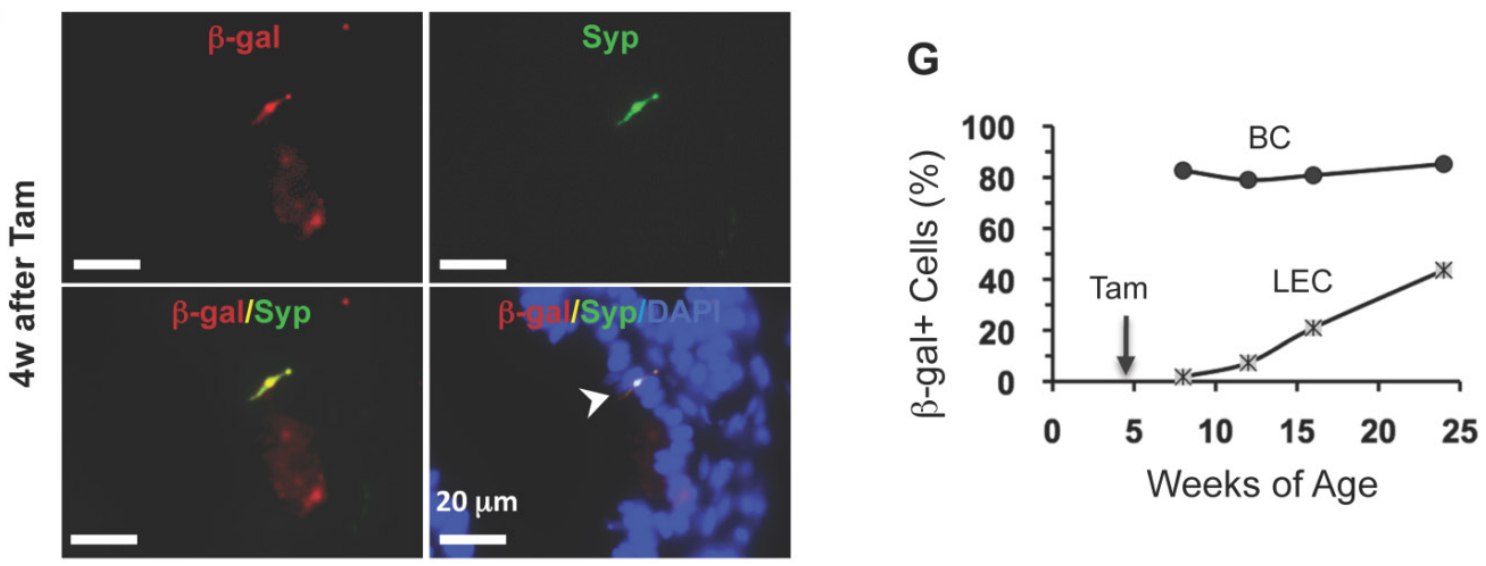

Figure 5. p63+ BC differentiation into BCs, LECs and NECs in adult prostates. A. Schematic of the lineage tracing experiments during prostate homeostasis in the adult p63-CreERT2×Rosa26R mice. B and C. X-gal-stained prostate tissues at 9 and I 2 weeks of age. The blue signal indicates $\beta$-gal+ cells in basal (panel B) and in both basal and luminal (panel C) layers. D and E. Unmerged and merged images of double immunofluoresent staining for $\beta$-gal and K5 or K8 on the prostatic sections from mice at weeks I and 4 after tamoxifen treatment. Arrowheads indicate double positive cells. F. Unmerged and merged images of double immunofluorescent staining for $\beta$-gal and Syp on the prostatic sections from mice at weeks I and 4 after tamoxifen treatment. The arrowhead indicates a double positive NEC. G. The percentage of $\beta$-gal+ BCs to total BCs and the percentage of $\beta$-gal+ LECs to total LECs at the indicated ages of p63-CreERT2×Rosa26R mice. Enhanced tamoxifen treatment was administrated to 4-week-old mice and prostate X-gal staining was performed at 8, 12, 16 and 24 weeks of ages. Data were obtained from 3 mice at each age point. 

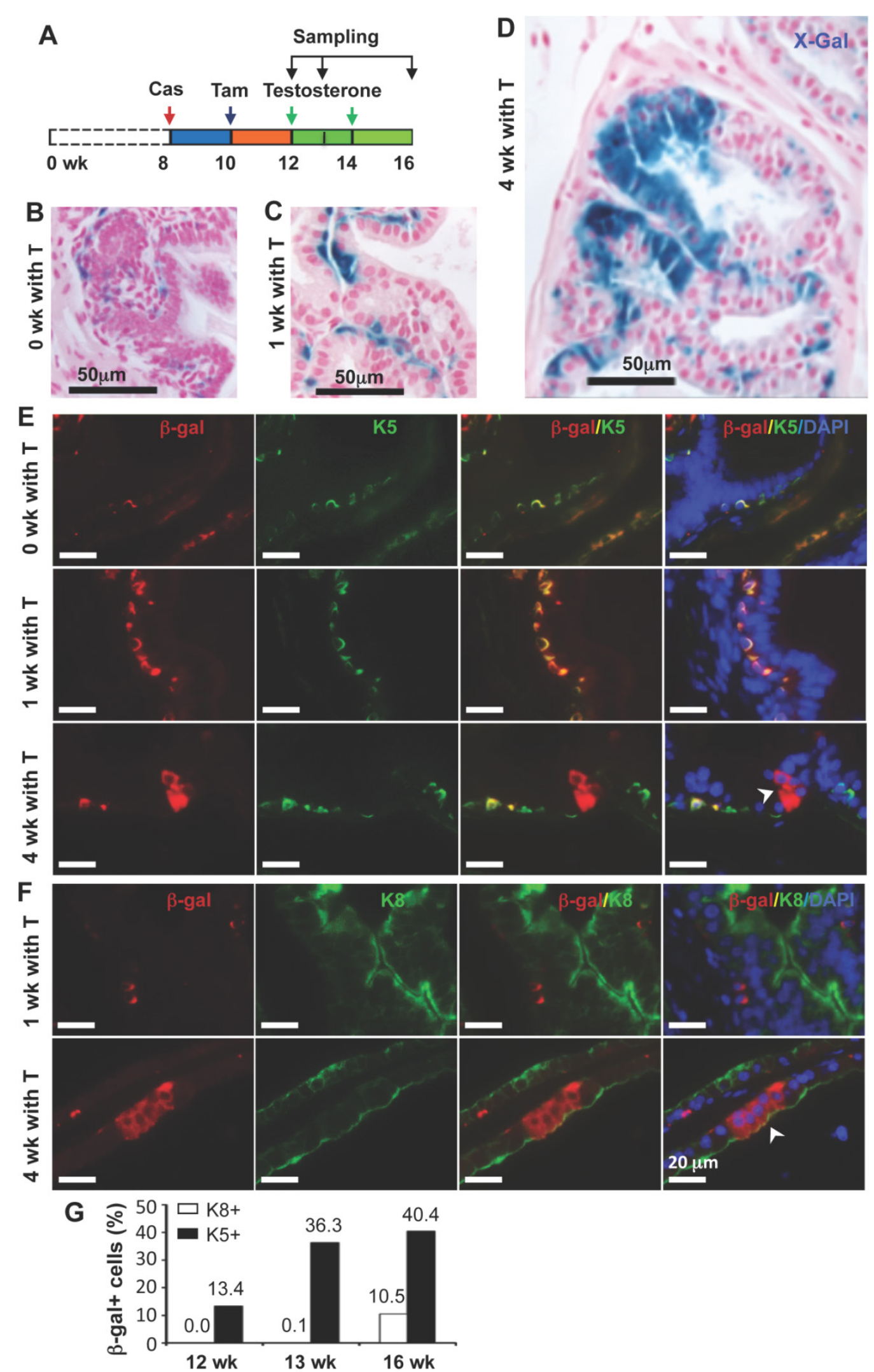

Figure 6. Differentiation of $\beta$-gal-labeled p63+ BCs into BCs and LECs during prostate regeneration. A. Schematic of the lineage tracing analysis during testosterone (T)-stimulated prostate regeneration in castrated CreERT2×Rosa26R mice. Cas, castration; wk, mouse ages in weeks. B-D. X-gal staining (blue) of prostate tissues at I2, 13 and 16 weeks of age, respectively. E. Unmerged and merged images of double fluorescent staining for $\beta$-Gal and K5 on the prostate sections from mice without T treatment $(0$ wk with $T)$ and with $T$ treatment for I and 4 weeks. Note the increased number of $\beta$-gal+/K5+ BCs at I week of $T$ treatment versus that at 0 week of $T$ treatment, which suggests new BCs derived from previous BCs. The arrowhead indicates $\beta$-gal+/K5- LECs. F. Unmerged and merged images of double fluorescent staining for $\beta$-gal and K8 on the prostate sections from mice with T treatment for I and 4 weeks. The arrowhead indicates the $\beta$-gal+/K $8+$ LECs. G. Percentages of $\beta$-gal+/K $8+$ to total K8+ cells and $\beta$-gal+/K5+ to total $\mathrm{K} 5+$ cells at 12,13 and 16 weeks of ages as indicated in panel $\mathrm{A}$. 


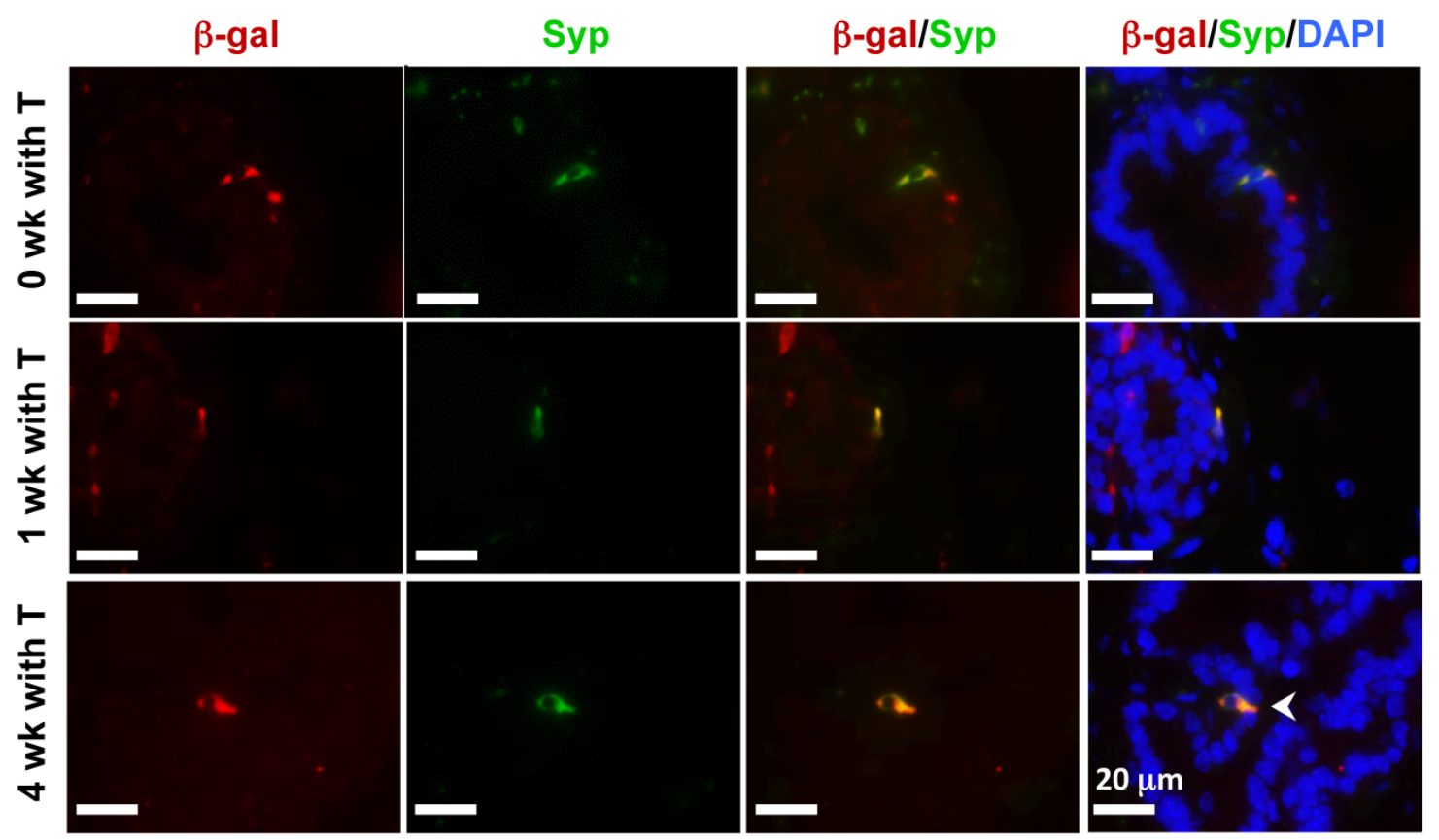

Figure 7. Androgen-independent differentiation of $\mathrm{p} 63+\mathrm{BC}$ s into NECs during prostate regression and regeneration. The $\mathrm{p} 63-\mathrm{CreERT} 2 \times \mathrm{Rosa} 26 \mathrm{R}$ mice were castrated at 8 weeks of age and treated with tamoxifen at 10 weeks of age and with T from 12 to 16 weeks of age (refer to Fig. 6A). Double immunofluorescent staining for $\beta$-gal and Syp were performed on the prostate sections from mice without $T$ treatment at 12 weeks of age $(0$ week with $T)$ and with $T$ treatment for $I$ and 4 weeks as indicated.

\section{Discussion}

A great effort has been devoted to identify stem cells in the prostatic epithelium because of their relevance to postnatal morphogenesis, and adult epithelial homeostasis and carcinogenesis. Initial studies using in vitro and ex vivo models showed that the epithelial cells, especially, the basal cell population in the adult prostate contain multipotent stem cells [16]. However, several recent in vivo studies reported controversial results regarding the identities of these stem cells in the basal cell layer. Ousset et al. demonstrated that the K14+ BCs marked at two weeks of age could efficiently differentiate into other types of epithelial cells [8], which is consistent with our results from tracing the $\mathrm{p} 63+\mathrm{BCs}$ at the same stage. In contrast to the stage of postnatal development, Choi et al. showed that the $\mathrm{K} 14+\mathrm{BCs}$ marked at five weeks of age only produced BCs but not LECs and NECs in the prostate [9], suggesting that the $\mathrm{K} 14+\mathrm{BCs}$ in the adult prostate are not stem cells. Lu et al. labeled the $\mathrm{K} 5+$ BCs in the mouse prostate at 5-6 weeks of age and observed that only $2 \%$ of LECs were derived from the labeled K5+ BCs after 17 weeks [18]. Furthermore, Wang et al. marked the K5+ BCs in 8-week-old adult mice, and found that almost no marked LEC was generated from these BCs under normal physiological condition, only $0.03 \%$ of marked LECs were generated from these BCs after one regression-regeneration cycle, and only $3.4 \%$ of marked LECs were generated even after five regression-regeneration cycles [10].
Given the inefficient differentiation and the age-dependent reduction of differentiation efficiency from the $\mathrm{K} 5+\mathrm{BC}$ to LECs, the $\mathrm{K} 5+\mathrm{BCs}$ are unlikely the majority of stem cells in the adult prostate. In relevance to the $\mathrm{K} 5+\mathrm{BC}$-tracing model, another previous study showed the castration-resistant Nkx3.1-expressing LECs (CARNs) could generate some of the LECs, BCs and NECs in the regression-regeneration cycles [19]. It is possible that the marked $\mathrm{K} 5+\mathrm{BCs}$ in the adult prostate are intermediate progenitor cells, which become some of the CARNs and subsequently differentiate into other types of epithelial cells during the regression-regeneration cycles. Intermediate progenitor cells express relatively low levels of both BC markers such as K5 and K14 and LEC markers such as K8 and K18. These intermediate cells have been considered as differentiating cells from BC compartment to LEC compartment many years ago [20-22].

Our results support the idea that the marked p63+ BCs efficiently differentiate into all of the three types of epithelial cells including LECs, BCs and NECs in the developing, adult and regenerating prostates. In comparison with previous studies, our study demonstrates, for the first time, that the p63+ BCs can generate NECs and can very efficiently develop into LECs in the adult prostate. The identification of the seven $B C$ subpopulations by the different expression fingerprints of p63, K5 and K14 should help to explain the discrepancies among the genetic models tracing the $\mathrm{K} 14+, \mathrm{K} 5+$ or $\mathrm{p} 63+\mathrm{BC}$ lineage. We 
found that $96.8 \%$ of total BCs and $96.0 \%$ of p63+ BCs express $\mathrm{K} 14$ and/or K5, indicating the majority of BCs or p63+ BCs are not stem cells in the adult prostate based on the reported lineage-tracing data from tracing the $\mathrm{K} 14+$ and the $\mathrm{K} 5+\mathrm{BCs}[8,9,19]$. Importantly, we identified a small BC subpopulation $(\sim 3.2 \%)$ that only expresses p63 but not K14 and K5. This finding suggests that the marked p63+ BCs include a unique $\mathrm{BC}$ subpopulation that has never been linage-traced previously and may be responsible for the major origin of LECs, NECs and BCs in the adult prostate.

\section{Acknowledgements}

We thank Souling Zhou for experimental assistance. We also thank the BCM Transgenic Mouse Core led by Dr. DeMayo for microinjection of mouse ES cells. This work was partially supported by $\mathrm{NIH}$ grants DK058242, CA112403 and DK059820 and a Cancer Prevention and Research Institute of Texas grant RP120732-P5.

This work was partially supported by $\mathrm{NIH}$ grants DK058242, CA112403 and DK059820 and a Cancer Prevention and Research Institute of Texas grant RP120732-P5.

\section{Competing Interests}

The authors have declared that no competing interest exists.

\section{References}

1. Hayward SW, Cunha GR. The prostate: development and physiology. Radiol Clin North Am. 2000; 38: 1-14.

2. Kasper S. Exploring the origins of the normal prostate and prostate cancer stem cell. Stem Cell Rev. 2008; 4: 193-201.

3. Goto K, Salm SN, Coetzee S, et al. Proximal prostatic stem cells are programmed to regenerate a proximal-distal ductal axis. Stem Cells. 2006; 24: $1859-68$

4. Lawson DA, Xin L, Lukacs RU, et al. Isolation and functional characterization of murine prostate stem cells. Proc Natl Acad Sci U S A. 2007; 104: 181-6.

5. Goldstein AS, Huang J, Guo C, et al. Identification of a cell of origin for human prostate cancer. Science. 2010; 329: 568-71.

6. Blackwood JK, Williamson SC, Greaves LC, et al. In situ lineage tracking of human prostatic epithelial stem cell fate reveals a common clonal origin for basal and luminal cells. J Pathol. 2011; 225: 181-8.

7. Gaisa NT, Graham TA, McDonald SA, et al. Clonal architecture of human prostatic epithelium in benign and malignant conditions. J Pathol. 2011; 225: $172-80$

8. Ousset M, Van Keymeulen A, Bouvencourt G, et al. Multipotent and unipotent progenitors contribute to prostate postnatal development. Nat Cell Biol. 2012; 14: 1131-8.

9. Choi N, Zhang B, Zhang L, et al. Adult murine prostate basal and luminal cells are self-sustained lineages that can both serve as targets for prostate cancer initiation. Cancer Cell. 2012; 21: 253-65.

10. Wang ZA, Mitrofanova A, Bergren SK, et al. Lineage analysis of basal epithelial cells reveals their unexpected plasticity and supports a cell-of-origin model for prostate cancer heterogeneity. Nat Cell Biol. 2013; 15: 274-83.

11. Liu Z, Zhou S, Liao L, et al. Imjd1a demethylase-regulated histone modification is essential for cAMP-response element modulator-regulated gene expression and spermatogenesis. J Biol Chem. 2010; 285: 2758-70.

12. Liu Z, Liao L, Zhou S, et al. Generation and validation of a mouse line with a floxed SRC-3/AIB1 allele for conditional knockout. Int J Biol Sci. 2008; 4: 202-7.

13. Farley FW, Soriano P, Steffen LS, et al. Widespread recombinase expression using FLPeR (flipper) mice. Genesis. 2000; 28: 106-10.

14. Soriano P. Generalized lacZ expression with the ROSA26 Cre reporter strain. Nat Genet. 1999; 21: 70-1.

15. Xin L, Ide $\mathrm{H}, \mathrm{Kim} \mathrm{Y}$, et al. In vivo regeneration of murine prostate from dissociated cell populations of postnatal epithelia and urogenital sinus mesenchyme. Proc Natl Acad Sci U S A. 2003; 100 Suppl 1: 11896-903.
16. Xin L, Lukacs RU, Lawson DA, et al Self-renewal and multilineage differentiation in vitro from murine prostate stem cells. Stem Cells. 2007; 25: 2760-9.

17. Pignon JC, Grisanzio C, Geng Y, et al. p63-expressing cells are the stem cells of developing prostate, bladder, and colorectal epithelia. Proc Natl Acad Sci U S A. 2013; 110: 8105-10.

18. Lu TL, Huang YF, You LR, et al. Conditionally ablated Pten in prostate basal cells promotes basal-to-luminal differentiation and causes invasive prostate cancer in mice. Am J Pathol. 2013; 182: 975-91.

19. Wang X, Kruithof-de Julio M, Economides KD, et al. A luminal epithelial stem cell that is a cell of origin for prostate cancer. Nature. 2009; 461: 495-500.

20. Verhagen AP, Aalders TW, Ramaekers FC, et al. Differential expression of keratins in the basal and luminal compartments of rat prostatic epithelium during degeneration and regeneration. Prostate. 1988; 13: 25-38.

21. Xue Y, Smedts F, Debruyne FM, et al. Identification of intermediate cell types by keratin expression in the developing human prostate. Prostate. 1998; 34: 292-301.

22. van Leenders GJ, Gage WR, Hicks JL, et al. Intermediate cells in human prostate epithelium are enriched in proliferative inflammatory atrophy. Am J Pathol. 2003; 162: 1529-37. 